In der pädagogischen und therapeutischen Arbeit in der Natur fehlt oft der Theorie-Praxis-Bezug bzw. eine wissenschaftliche Grundlage. Das vorliegende Werk soll Fachkräften eine wissenschaftliche Grundlage für die Arbeit in ihrer Praxis bieten. Fachkräfte arbeiten in dem Naturraum, weil sie der Meinung sind und auch in ihrer Arbeit immer wieder darin bestärkt werden, dass die Arbeit mit dem Naturraum ihrem Klientel 'gut tut‘ bzw. einen positiven Einfluss auf dessen Entwicklung hat. Das vorliegende Handbuch stellt dar, inwieweit Natur im Bereich der Pädagogik und Therapie genutzt werden kann, um Entwicklungspotentiale in den unterschiedlichen Entwicklungsphasen eines Menschen weiter voranzutreiben. Hierbei bezieht sich der Autor nicht nur auf die Förderung der Entwicklung sondern auch auf die Förderung von Gesundheit und Bildung.

Späker bietet in seinem Werk eine Zusammenführung diverser Studien in den jeweiligen Fachdisziplinen, die die positiven Wirkungen der Arbeit im Naturraum bzw. der einzelnen Fachdisziplinen aufweisen und bestätigen. Diese Studien haben zu sehr ähnlichen Ergebnissen geführt und bestätigen somit die Möglichkeiten und Potentiale der Nutzung der Arbeit im Naturraum. Diese Zusammenführung ist ein Schritt dahingehend, die Theorie-Praxis-Lücke etwas weiter zu schließen, und bietet den Fachkräften eine Argumentationsgrundlage gegenüber Vorgesetzten und Finanziers. Des Weiteren können die Inhalte des vorliegenden Werkes Fachkräfte in ihrer Arbeit inhaltlich bestätigen.

Späker schlägt einen großen Bogen von der Begriffsbestimmung "Natur" über den Einfluss von Naturräumen auf die einzelnen Lebensabschnitte eines Menschen und über die Darstellung der einzelnen Fachdisziplinen und $\mathrm{Na}$ tur-Erfahrungsfelder zu handlungsleitenden Grundlagen für die Praxis. Er schafft es, diesen weiten Bogen übersichtlich, aufeinander aufbauend und strukturiert zusammengefasst darzustellen. Der Autor gibt einen guten und komprimierten Einblick in die einzelnen Fachdisziplinen und in die $\mathrm{Na}$ tur-Erfahrungsfelder. Für detailliertere Einblicke oder gestaltende bzw. ausdifferenzierte Ideen zur Gestaltung von Arbeitsinhalten im Naturraum in den einzelnen Fachdisziplinen sollten sich die LeserInnen in weiterführende Literatur einarbeiten. Hierfür finden sich Verweise in den einzelnen Kapiteln bzw. im Literaturverzeichnis. Das Werk richtet sich an Fachkräfte, die im Naturraum arbeiten und entsprechende Vorkenntnisse haben, oder an Menschen, die einen Überblick über die verschiedenen möglichen pädagogischen und therapeutischen Handlungsfelder im Naturraum gewinnen möchten.

Das Handbuch ist verständlich und gut lesbar geschrieben, Schaubilder veranschaulichen komplexere Zusammenhänge und Zusammenfassungen am jeweiligen Kapitelende erleichtern ein schnelles Nachschlagen bzw. bieten einen guten Überblick. Des Weiteren wird dargelegt, wie die einzelnen Fachdisziplinen gegeneinander abzugrenzen sind, welche einzelnen Schwerpunktsetzungen vorliegen und welche Zielsetzungen in den einzelnen Gebieten bestehen.

Neben den positiven Auswirkungen von der Arbeit in und mit der Natur weist Späker auch die Grenzen auf, die eine solche Arbeit mit sich bringen kann. Die in seinem Werk aufgeführten

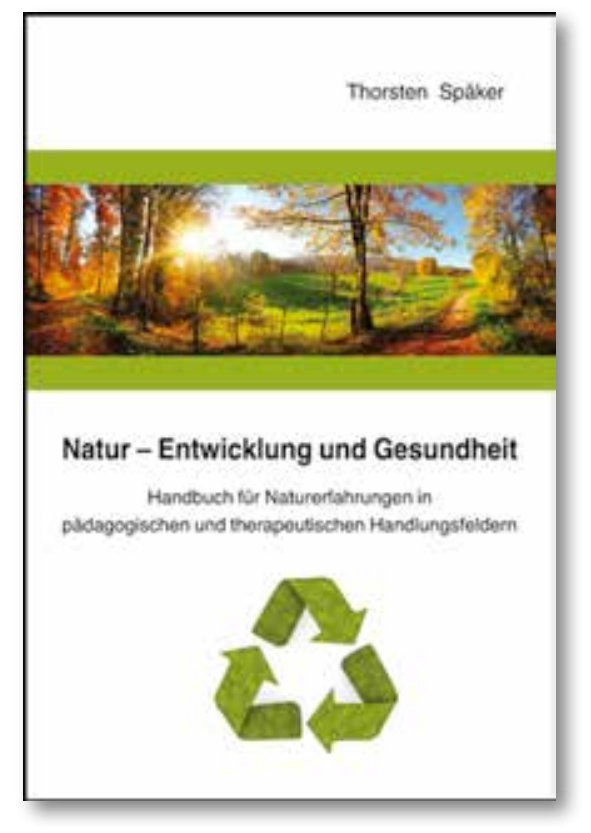

Grenzen beziehen sich vor allem auf Ängste, Ekel, negative Vorerfahrungen und Überforderung der Klientel und einen oftmals erhöhten organisatorischen und finanziellen Aufwand. In diesem Kapitel hätte Späker ausführen können, inwieweit trotz dieser Grenzen bzw. mit diesen Einschränkungen auf eine Arbeit in der Natur hingearbeitet werden kann, z.B. mithilfe des LernKomfortzonenmodells (117), Möglichkeiten der Akquise von finanziellen Mitteln und kleinschrittige Zugänge zur Arbeit / zu Aufenthalten in der Natur zu schaffen.

\section{Anke Sommerhäuser}

DOI 10.2378/mot2019.artogd 